\title{
BMJ Open Chinese herbal medicine for patients with vascular cognitive impairment no dementia: protocol for a systematic review
}

Mei Feng, ${ }^{1,2,3}$ Jingmin Lu, ${ }^{3}$ Brian H May, ${ }^{4}$ Shaonan Liu, ${ }^{1,2,3}$ Xinfeng Guo, ${ }^{1,2,3}$ Anthony Lin Zhang, ${ }^{4}$ Charlie Changli Xue, ${ }^{2,3,4}$ Chuanjian Lu ${ }^{2,3,4}$

To cite: Feng M, Lu J, May BH, et al. Chinese herbal medicine for patients with vascular cognitive impairment no dementia: protocol for a systematic review. BMJ Open 2016;6:e10295.

doi:10.1136/bmjopen-2015010295

- Prepublication history and additional material is available. To view please visit the journal (http://dx.doi.org/ 10.1136/bmjopen-2015010295)

Received 19 October 2015 Revised 26 January 2016 Accepted 15 February 2016

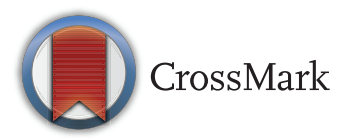

For numbered affiliations see end of article.

Correspondence to Professor Chuanjian Lu; luchuanjian888@vip.sina.com and Professor Charlie Changli Xue; charlie.xue@rmit.edu.au

\section{ABSTRACT}

Introduction: The aim of this systematic review is to assess the efficacy and safety of Chinese herbal medicine for the treatment of patients with vascular cognitive impairment but no dementia.

Methods and analysis: We will perform a comprehensive retrieval in the following electronic databases: PubMed, Cochrane Library, EMBASE, CINAHL, Chinese Biomedical Literature Service System (SinoMed), China National Knowledge Infrastructure (CNKI), Chinese Scientific Journals Database (VIP), Wan-fang database and other sources. After screening the studies, the methodological quality of all included trials will be assessed according to the risk of bias instrument provided by the Cochrane Collaboration. A meta-analysis of randomised controlled trials will be conducted using RevMan V.5.3 software. Funnel plot analysis and Egger's test will be used to assess publication bias, if possible. The quality of evidence will be assessed by the GRADE system.

Dissemination: This systematic review will be disseminated in a peer-reviewed journal and a relevant conference presentation.

Trial registration number: PROSPERO CRD 42015023682.

\section{INTRODUCTION}

As the population ages and survival from vascular disease are improved, cognitive dysfunction and dementia caused by cerebrovascular disease (CVD) have become prevalent. $^{1} \quad$ Recently, vascular cognitive impairment (VCI) has been introduced to describe the entire spectrum of cognitive dysfunction-ranging from cognitive impairment without dementia to dementiaattributable to all forms of CVD. ${ }^{12}$ There are three subtypes of VCI: VCI no dementia (VCIND), vascular dementia (VaD) and mixed $\mathrm{VaD}$ and Alzheimer's disease (AD). ${ }^{3}$ Among them, VCIND is characterised by early or mild cognitive impairment due to

\section{Strengths and limitations of this study}

- This study is a comprehensive, objective and normative systematic review on the efficacy and safety of Chinese herbal medicine for vascular cognitive impairment in participants without dementia.

- Study screening, data extraction and assessment of the risk of bias will be conducted independently by two researchers.

- Currently, there is a lack of internationally recognised diagnostic criteria in vascular cognitive impairment without dementia. Therefore, subgroup analyses will be carried out to explore any sources of heterogeneity.

cerebrovascular injuries with an insidious nature. ${ }^{4}$ It may be considered to be the prodromal stage of $\mathrm{VaD},{ }^{5}$ with occurrence in $26.9 \%$ of patients approximately 3 months after stroke onset. ${ }^{6}$ A cohort study which included 149 participants with VCIND showed that $46 \%$ cases were observed to develop dementia, and $52 \%$ of these cases died within 5 years. ${ }^{7}$ Another communitybased, cross-sectional study in China also reported that the overall prevalence of poststroke cognitive impairment (PSCI) was $80.97 \%$ (95\% CI $77.82 \%$ to $84.11 \%$ ), while that of non-dementia PSCI (PSCI-ND) and post-stroke VaD was $48.91 \%$ (95\% CI $44.91 \%$ to $52.92 \%$ ) and $32.05 \%$ (95\% CI $8.32 \%$ to $35.79 \%)$, respectively. ${ }^{8}$

Fortunately, VCIND is potentially treatable and preventable. ${ }^{3}$ Early diagnosis and appropriate interventions may contribute to the improvement of the prognosis. ${ }^{9}$ However, there are limited treatment options to improve cognition and function in VCI. ${ }^{1}$ Chinese herbal medicine (CHM), which has been used for thousands of years in China, has been considered promising for improving cognitive function. ${ }^{10} \mathrm{~A}$ meta-analysis of 
CHM for VaD showed that there were significant benefits associated with CHM groups in terms of effective rate, Mini-Mental State Examination (MMSE) scores and Hasegawa Dementia Scale scores at the end of treatment. Furthermore, fewer adverse events occurred in the treatment group. In the light of this evidence, CHM appears to be safe and effective for $\mathrm{VaD}^{11} \mathrm{~A}$ meta-analysis which focused on the efficacy and safety of CHM for VCIND indicated that CHM could improve patients' cognitive and behavioural functions compared to the treatment with Western medicine. ${ }^{12}$ However, the information source was limited to Chinese medical databases and there was no flow chart to show the study screening process. Therefore, we aim to carry out a more comprehensive and normative systematic review to provide objective and scientific evidence on CHM for the treatment of VCIND.

\section{METHODS AND ANALYSIS}

\section{Inclusion and exclusion criteria for study selection}

Types of studies

Randomised controlled trials (RCTs) which used CHM or a combination of CHM and routine pharmacotherapy as treatment measures will be eligible. Language will be limited to English and Chinese.

\section{Types of participants}

1. No internationally recognised diagnostic criteria currently exist and no laboratory tests are available for VCIND diagnosis. ${ }^{13} 14$ Therefore, this study will include patients who were diagnosed with VCIND based on diagnostic criteria used in international clinical trials ${ }^{15-17}$ or Chinese expert consensus. ${ }^{18-20}$

2. The study will include all types of patients regardless of their age, sex, ethnicity, education or economic status and whether or not they were outpatients or inpatients.

3. Patients should have acquired cognitive impairment, but not meet the diagnostic criteria for any type of dementia. Furthermore, their cognitive impairment was judged to have vascular risk factors or CVD (including clinical features or radiographic features).

\section{Types of interventions}

Studies reporting any type of CHM treatment will be included. CHM could be used alone or combined with routine pharmacotherapy. Studies where the control group is different from the pharmacotherapy in the intervention group will be excluded. Trials in which the intervention group includes any other Chinese medicine therapies (eg, acupuncture, moxibustion, massage, taichi, etc) will be excluded.

\section{Types of comparators}

Comparators will include placebo control, no treatment, routine pharmacotherapy, routine care and other conventional treatments.

\section{Primary outcome assessments}

Primary outcome measures for assessment should include at least one of the internationally recognised evaluation scales, which refer to one of the following aspects: cognitive function, activities of daily living, behavioural and psychological symptoms of dementia (BPSD) or global evaluation scales (table 1).

\section{Secondary outcome assessments}

The secondary outcome assessments will be the incidence and severity of adverse events.

\section{Search methods for identification of studies \\ Electronic searches}

We will perform a comprehensive retrieval for studies that evaluate the efficacy and safety of CHM in patients with VCIND in major Chinese and English medical databases including PubMed, Cochrane Library, EMBASE, CINAHL, Chinese Biomedical Literature Service System (SinoMed), China National Knowledge Infrastructure (CNKI), Chinese Scientific Journals Database (VIP) and Wan-fang database from their respective inceptions to July 2015. The following subject terms and key words

\begin{tabular}{|c|c|}
\hline Outcome categories & Outcome measures \\
\hline Cognitive function & $\begin{array}{l}\text { MMSE (Mini-Mental } \\
\text { Status Examination) } \\
\text { HDS (Hasegawa } \\
\text { Dementia Scale) } \\
\text { ADAS-cog (Alzheimer's } \\
\text { disease assessment } \\
\text { scale-cognitive) } \\
\text { MoCA (Montreal Cognitive } \\
\text { Assessment) } \\
\text { WMS (Wechsler Memory } \\
\text { Scale) }\end{array}$ \\
\hline Activities of daily living & $\begin{array}{l}\text { Activities of daily living } \\
\text { ADCS-ADL (Alzheimer } \\
\text { disease cooperative } \\
\text { study-ADL inventory) } \\
\text { FAQ (Functional activity } \\
\text { questionnaire) }\end{array}$ \\
\hline $\begin{array}{l}\text { Behavioural and } \\
\text { psychological symptoms of } \\
\text { dementia (BPSD) }\end{array}$ & $\begin{array}{l}\text { NPI (neuropsychiatric } \\
\text { inventory) } \\
\text { HAMD (Hamilton } \\
\text { depression scale) } \\
\text { CSDD (Cornell scale for } \\
\text { depression in dementia) }\end{array}$ \\
\hline Global evaluation & $\begin{array}{l}\text { CDR (clinical dementia } \\
\text { rating) } \\
\text { CIBIC-plus (clinicians' } \\
\text { interview-based } \\
\text { impression of } \\
\text { change-plus) } \\
\text { GDS (global deterioration } \\
\text { scale) }\end{array}$ \\
\hline
\end{tabular}


will be used in the search: ('vascular cognitive impairment' OR 'mild cognitive impairment' OR 'post-stroke cognitive impairment' OR 'VCI') and ('Medicine, Chinese Traditional' OR 'Drugs, Chinese Herbal' OR 'herbal medicine' OR 'Chinese traditional medicine' OR 'Traditional Chinese medicine'). Chinese translations of these search terms will be utilised to search the Chinese databases. The search strategy for PubMed is given in table 2 and detailed steps are shown in online supplementary appendix 1 .

\section{Other sources}

Studies from other sources will also be obtained from the following sources:

1. Google scholar (http://scholar.google.com) and Baidu scholar (http://xueshu.baidu.com/);

2. Clinical Trials.gov (http://www.clinical trails.gov) and Chinese Clinical Trial Registry (http://www.chictr. org $/ \mathrm{cn} /$ );

3. The reference lists of the retrieved articles.

\section{Data extraction and quality evaluation}

EndNote X6 software will be used to manage the literature and the process of removing duplications. A data extraction EXCEL table will be designed which includes the data on age, sex, duration, education level, lifestyle (eg, smoking, alcohol consumption), vascular risk factors (eg, hypertension, hyperlipidaemia, diabetes), medical comorbidities, concurrent medications (eg, antiplatelet aggregation drugs, antihypertensive drugs, hypoglycaemic drugs), randomisation, blinding, interventions, comparators, outcomes, study dropouts, the incidence and details of adverse effects. Two researchers (MF and JL) will extract and double-check the data from the included articles. Any discrepant data will be reviewed by discussion with another team member (SL). The details of the study selection will be shown in a PRISMA flow chart (figure 1).

\begin{tabular}{ll} 
Table 2 & Search strategy \\
\hline No & Search in the PubMed database \\
\hline 1 & vascular cognitive impairment \\
2 & mild cognitive impairment \\
3 & post-stroke cognitive impairment \\
4 & VCl \\
5 & or $1-4$ \\
6 & Medicine, Chinese Traditional \\
7 & Drugs, Chinese Herbal \\
8 & herbal medicine \\
9 & Chinese traditional medicine \\
10 & Traditional Chinese medicine \\
11 & or $6-10$ \\
12 & 5 and 11 \\
\hline
\end{tabular}

This search strategy will be modified as required for other electronic databases.

\section{Risk of bias assessment of included studies}

The methodological quality of all included trials will be assessed by a 'risk of bias assessment tool' according to the Cochrane Handbook 5.1 by two independent researchers (MF and JL). Any different views will be discussed with another team member (SL). The risk of bias in the included studies will be evaluated according to the eight domains: randomisation sequence generation, randomisation allocation concealment, blinding of participants, blinding of personnel, blinding of outcome assessors, incomplete outcome data, selective outcome reporting and other sources of bias. Since many reports of RCTs published in Chinese journals lacked an adequate description of randomisation sequence generation and allocation concealment ${ }^{21}$ we will retrieve related protocols in clinical trials registration websites and contact first author/s or corresponding author/s through email or telephone for further details of these two domains.

\section{Strategy for data synthesis and statistical analysis}

We will use RevMan V.5.3 software for data analysis. For continuous data collected using the same measurement scale, we will calculate weighted mean difference and 95\% CIs. For continuous data collected using similar measurement scales for the same outcome, we will calculate standardised mean differences with $95 \%$ CIs. For dichotomous outcomes, we will use risk ratio with $95 \%$ $\mathrm{CI}$ and $\mathrm{p}$ values to assess the efficacy and safety of CHM. A standard $\chi^{2}$ statistic and $\mathrm{I}^{2}$ statistic will be used to test heterogeneity between groups. Data will be analysed with a fixed-effect model if no statistical heterogeneity was observed between subgroups $\left(p \geq 0.1, \mathrm{I}^{2} \leq 50 \%\right)$. In the presence of heterogeneity $\left(\mathrm{p}<0.1, \mathrm{I}^{2}>50 \%\right)$, a random-effect model will be used and the possible causes of heterogeneity will be examined.

\section{Dealing with missing data or unclear scale type}

We will attempt to contact the authors of included studies in which there are missing data or unclear sources of the evaluation scales through email or telephone. For missing data, if we fail to obtain sufficient data after contacting authors, an intention-to-treat analysis will be performed if feasible.

\section{Planned subgroup analyses}

Where possible, we will perform subgroup analyses based on different demographic characteristics or vascular risk factors, severity of included patients, different disease durations, diagnostic criteria, intervention type (CHM or CHM in combination with routine pharmacotherapy), routes of administration, dosage, preparations and ingredients of CHM interventions and controls.

\section{Sensitivity analysis}

Where feasible, we will carry out sensitivity analyses to investigate the robustness of the study conclusions, such as grouping studies by different levels of the methodological quality. 
Figure 1 Flow diagram of selection process.

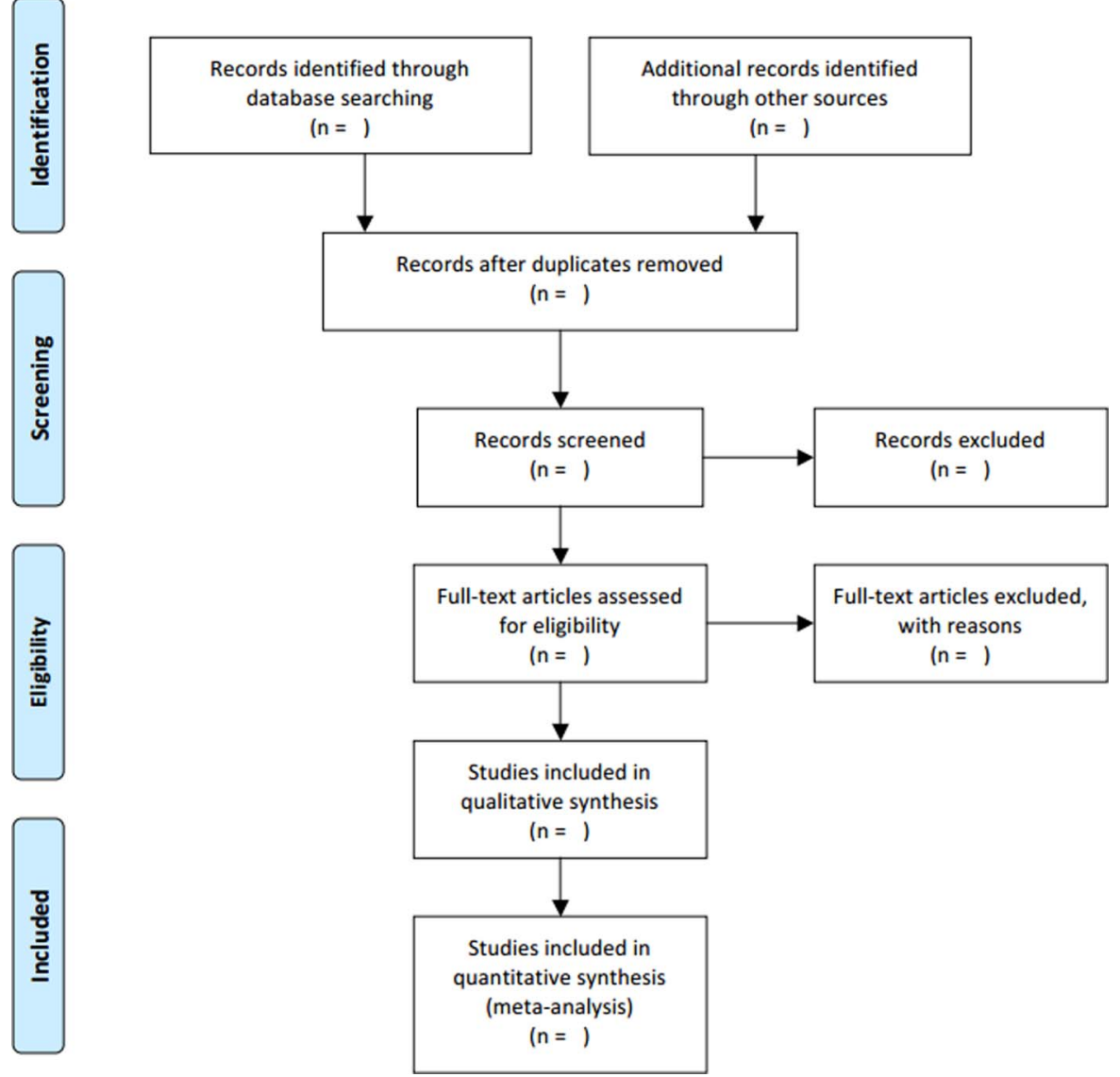

\section{Assessment of publication biases}

We will use funnel plots in RevMan V.5.3 and Egger's test in Stata 11.0 to detect publication bias if more than 10 studies are included in the meta-analysis.

\section{Assessment of quality of evidence}

The Cochrane Collaboration Network GRADE (The Grading of Recommendations Assessment Development and Evaluation) will be used to assess the results in this systematic review. We will create a Summary of Finding table in http://www.guidelinedevelopment.org/.

\section{Ethics and dissemination}

Ethical approval will not be required for this systematic review because there are no concerns regarding the patient's privacy. The results will be disseminated in a peer-reviewed journal and presented at a relevant conference.

\section{DISCUSSION}

VCIND belongs to the categories of Jianwang, Shanwang, Daibing in traditional Chinese medicine. ${ }^{22}$ CHMs have been cited for treating memory disorders in the classical herbal literature for many centuries, ${ }^{23}$ and the results of preclinical and clinical studies suggest that CHM could improve cognitive decline. ${ }^{24} 25$ For example, Ginkgo biloba extract EGb 761 is the most extensively clinically tested Chinese herbal-based substance for cognitive impairment and dementia and meta-analytic evidence suggests that EGb 761 provides benefit for stabilising or slowing decline in cognition of people with cognitive impairment and dementia. ${ }^{26}$ Huperzine $\mathrm{A}$ is another Chinese herbal derivative which has been authorised for treating $\mathrm{AD}$ and benign memory deficits since 1994 in China. ${ }^{27}$ As an alkaloid isolated from the Chinese herb Huperzia serrata, Huperzine A can selectively inhibit acetylcholinesterase activity and thus facilitate the increase in acetylcholine level in the brain, thereby improving cognitive function in patients with dementia. ${ }^{28}$ Meta-analysis also shows that Huperzine A could significantly improve the MMSE and ADL scores of patients with $\mathrm{AD}$ and $\mathrm{VD}$ with a good tolerance. ${ }^{28}$ However, there is, as yet, no overall assessment of the clinical evidence regarding CHM interventions for VCIND in evidence-based medicine.

Thus, we plan to conduct this meta-analysis to evaluate the efficacy and safety of CHM therapies for patients with VCIND. We hope that this review will provide evidence to assist clinicians and patients' decision-making process when dealing with VCIND. However, no internationally recognised VCIND diagnostic criteria exist currently. This will be a potential source of heterogeneity for the included study population and influence the extrapolation of conclusion. Therefore, subgroup analyses according to the characteristics of VCIND and different diagnosis criteria will need to be carried out. 
PRISMA-P (Preferred Reporting Items for Systematic review and Meta-Analysis Protocols) 2015 checklist $^{29}$ of this protocol is presented in online supplementary Appendix 2.

\section{Author affiliations}

${ }^{1}$ Evidence-based Medicine and Clinical research Service Group, Guangdong Provincial Hospital of Chinese Medicine, Guangzhou, China

${ }^{2}$ Guangdong Provincial Academy of Chinese Medical Sciences, Guangzhou, China

${ }^{3}$ The Second Clinical College, Guangzhou University of Chinese Medicine, Guangzhou, China

${ }^{4}$ China-Australia International Research Centre for Chinese Medicine, School of Health and Biomedical Sciences, RMIT University, Melbourne, Victoria, Australia

Contributors $\mathrm{CL}, \mathrm{CCX}, \mathrm{XG}$ and ALZ conceived and designed the study. The manuscript of this protocol was drafted by MF, JL and BHM and revised by $\mathrm{CL}$ and CCX. MF and JL designed the search strategies and will perform the search, screening and assessment of the risk of bias independently. MF and BHM will analyse and interpret the data. SL will arbitrate any disagreements during the review. All authors approved the final version of this protocol.

Funding This study is funded by a Project Grant from the Guangdong Provincial Science \& Technology Department and the Guangdong Provincial Academy of Chinese Medical Sciences (GPACMS) (Project Grant no. 2012A032500009). The study is partially supported by a Grant from the International Science \& Technology Cooperation Project of the Ministry of Science and Technology of China (Project Grant no. 2012DFA31760) and International Science and Technology Cooperation Project of TCM funded by the State Administration of TCM, China (Project Grant no.1601500000027 (11)).

Competing interests None declared.

Provenance and peer review Not commissioned; externally peer reviewed.

Data sharing statement No additional data are available.

Open Access This is an Open Access article distributed in accordance with the Creative Commons Attribution Non Commercial (CC BY-NC 4.0) license, which permits others to distribute, remix, adapt, build upon this work noncommercially, and license their derivative works on different terms, provided the original work is properly cited and the use is non-commercial. See: http:// creativecommons.org/licenses/by-nc/4.0/

\section{REFERENCES}

1. Ritter A, Pillai JA. Treatment of vascular cognitive impairment. Curr Treat Options Neurol 2015;17:367.

2. Levine DA, Langa KM. Vascular cognitive impairment: disease mechanisms and therapeutic implications. Neurotherapeutics 2011:8:361-73.

3. O'Brien JT, Erkinjuntti T, Reisberg B, et al. Vascular cognitive impairment. Lancet Neurol 2003;2:89-98.

4. Black SE. Vascular cognitive impairment: epidemiology, subtypes, diagnosis and management. $J R$ Coll Physicians Edinb 2011;41:49-56.

5. Stephan BC, Matthews FE, Khaw KT, et al. Beyond mild cognitive impairment: vascular cognitive impairment, no dementia (VCIND). Alzheimers Res Ther 2009;1:4.

6. Serrano S, Domingo J, Rodríguez-Garcia E, et al. Frequency of cognitive impairment without dementia in patients with stroke: a two-year follow-up study. Stroke 2007;38:105-10.

7. Wentzel C, Rockwood K, MacKnight C, et al. Progression of impairment in patients with vascular cognitive impairment without dementia. Neurology 2001;57:714-16.
8. Qu YJ, Zhuo L, Li N, et al. Prevalence of post-stroke cognitive impairment in China: a community-based, cross-sectional study. PLOS ONE 2015;10:e0122864.

9. Jiang B, Ding C, Yao G, et al. Polysomnographic abnormalities in patients with vascular cognitive impairment-no dementia. Sleep Med 2013;14:1071-5.

10. Xu X, Guo L, Tian G. Diabetes cognitive impairments and the effect of traditional Chinese herbs. Evid Based Complement Alternat Med 2013;2013:649396.

11. Qin X, Liu Y, Wu Y, et al. A meta-analysis of Chinese herbal medicines for vascular dementia. Neural Regen Res 2013;8:1685-92.

12. Shuang $X P, \operatorname{Tan} Z \mathrm{H}$, Chen $\mathrm{Q}$, et al. Efficacy and safety of TCM therapy for VCIND: a meta-analysis. Chin Arch Tradit Chin Med 2015;33:387-91.

13. Hachinski V, ladecola C, Petersen RC, et al. National Institute of Neurological Disorders and Stroke-Canadian Stroke Network vascular cognitive impairment harmonization standards. Stroke 2006;37:2220-41

14. Wang LP, Zhang XY, Liu N, et al. Comparison of integrated traditional Chinese and western medicine therapy on vascular cognitive impairment with no dementia. Genet Mol Res 2015;14:4896-902.

15. Ingles JL, Wentzel C, Fisk JD, et al. Neuropsychological predictors of incident dementia in patients with vascular cognitive impairment, without dementia. Stroke 2002;33:1999-2002.

16. Wentzel C, Darvesh S, MacKnight C, et al. Inter-rater reliability of the diagnosis of vascular cognitive impairment at a memory clinic. Neuroepidemiology 2000;19:186-93.

17. Neuropathology Group. Medical Research Council Cognitive Function and Aging Study. Pathological correlates of late-onset dementia in a multicentre, community-based population in England and Wales. Neuropathology Group of the Medical Research Council Cognitive Function and Ageing Study (MRC CFAS). Lancet 2001;357:169-75.

18. Experts consensus group of Chinese prevention and treatment of cognitive dysfunction. Experts' common understanding of Chinese prevention and treatment of cognitive dysfunction. Chin J Intern Med 2006;45:171-3.

19. Experts consensus group of Vascular cognitive impairment. Experts' common understanding of vascular cognitive impairment. Chin $J$ Intern Med 2007;46:1052-5.

20. Jia JP. Attention to early diagnosis and intervention of vascular cognitive impairment. Chin J Neurol 2005;38:4-6.

21. Wu T, Li Y, Bian Z, et al. Randomized trials published in some Chinese journals: how many are randomized?. Trials 2009;10:46.

22. Zhao LQ, Rao KH, Liu HS. Experience of Wendan decoction treating for vascular cognitive impairment, no dementia. Guangming J Chin Med 2014;29:2176-7.

23. May BH, Lu C, Lu Y, et al. Chinese herbs for memory disorders: a review and systematic analysis of classical herbal literature. $J$ Acupunct Meridian Stud 2013:6:2-11.

24. Ye S, Gu Y, Xu Y, et al. Bushen Huoxue decoction improves cognitive decline in rats with cerebral hypoperfusion. Mol Med Rep 2014:10:1635-41.

25. Liu JH, Chen J, Tan ZH, et al. Effects of dioscorea modified pill on cognitive impairment of patients with VCIND: an preliminary study of proton magnetic resonance spectroscopy. Zhonghua Yi Xue Za Zhi 2014;94:3075-8.

26. Solfrizzi V, Panza F. Plant-based nutraceutical interventions against cognitive impairment and dementia: meta-analytic evidence of efficacy of a standardized Gingko biloba extract. J Alzheimers Dis 2015;43:605-11.

27. Hao Z, Liu M, Liu Z, et al. Huperzine A for vascular dementia. Cochrane Database Syst Rev 2009;(15):CD007365.

28. Xing SH, Zhu CX, Zhang R, et al. Huperzine A in the treatment of Alzheimer's disease and vascular dementia: a meta-analysis. Evid Based Complement Alternat Med 2014;2014:363985

29. Shamseer L, Moher D, Clarke M, et al. Preferred reporting items for systematic review and meta-analysis protocols (PRISMA-P) 2015: elaboration and explanation. BMJ 2015;349:g7647. 